\title{
Stability, diffusion, and complex formation of beryllium in wurtzite GaN
}

\author{
Sukit Limpijumnong, Chris G. Van de Walle, and Jörg Neugebauer ${ }^{1}$ \\ Xerox PARC, Palo Alto, CA 94304, U.S.A. \\ ${ }^{1}$ Fritz-Haber-Institut, Berlin, Germany.
}

\section{ABSTRACT}

We have studied the properties of Be dopants in $\mathrm{GaN}$ using first principles calculations. Substitutional Be on a $\mathrm{Ga}$ site acts as an acceptor, but interstitial Be poses a potential problem because of its low formation energy and donor character. We study the diffusion of interstitial Be and find it to be highly anisotropic. We also study the formation of complexes between substitutional and interstitial $\mathrm{Be}$, and between substitutional $\mathrm{Be}$ and hydrogen. We have calculated the Be-H vibrational modes to aid in experimental identification of such complexes.

\section{INTRODUCTION}

Acceptor doping of nitride semiconductors still poses serious challenges. Magnesium can be incorporated up to about $10^{20} \mathrm{~cm}^{-3}$ before a saturation and subsequent decrease of the hole concentration sets in [1]. But Mg has a large ionization energy (210 meV, Ref. [2]), resulting in a low hole concentration at room temperature since only $\sim 1 \%$ of $\mathrm{Mg}$ are ionized. Previous computational studies that explored various candidate acceptors showed that $\mathrm{Be}$ is a viable acceptor due to its high solubility and low ionization energy compared to $\mathrm{Mg}[3,4]$. However, the small size of Be renders incorporation on interstitial sites ( $\mathrm{Be}_{\text {int }}$, which acts as a donor) almost as favorable as on the substitutional $\mathrm{Ga}$ site $\left(\mathrm{Be}_{\mathrm{Ga}}\right)$, where $\mathrm{Be}$ needs to reside in order to act as an acceptor. In order to assess the consequences of this behavior, we have carried out a comprehensive study of the stability and diffusivity of Be interstitials. In addition, we have examined complex formation, both between $\mathrm{Be}_{\mathrm{Ga}}$ and $\mathrm{Be}_{\mathrm{int}}$, and between $\mathrm{Be}_{\mathrm{Ga}}$ and hydrogen; the latter is known to play an important role in p-type doping of GaN. In the next sections, we will briefly discuss the theoretical approach and summarize our main results for stability and diffusivity of $\mathrm{Be}_{\text {int }}$. We will then focus on the details of vibrational modes of $\mathrm{Be}_{\mathrm{Ga}}-\mathrm{H}$ complexes, which we hope will serve as a signature for probing this complex experimentally.

\section{THEORETICAL APPROACH}

We use density-functional theory (DFT) in the local density approximation and ab initio norm-conserving pseudopotentials, with a plane-wave basis set. The effect of Ga $3 d$ states was included through the "nonlinear core correction" (nlcc), allowing us to use an energy cutoff of 40 Ry. The theoretical lattice constant of bulk GaN is used throughout, in order to avoid any spurious relaxations. We use a supercell geometry, consisting of several primitive unit cells of wurtzite GaN and containing one impurity. 32-, 72-, and 96-atom cells were used in this study. Convergence tests indicate that 96-atom cells yield formation energies converged to better than $0.1 \mathrm{eV}$. Such large supercells are computationally very demanding. In order to be able to perform calculations for a large number of configurations, particularly in the course of mapping out total-energy surfaces or studying vibrational properties, we used 32-atom supercells, which were found adequate for obtaining energy differences between different impurity positions in the lattice. Brillouin-zone integrations were carried out with $3 k$-points in the irreducible part of the zone. Additional details about the computational approach can be found in Refs. [4] and [5]. 


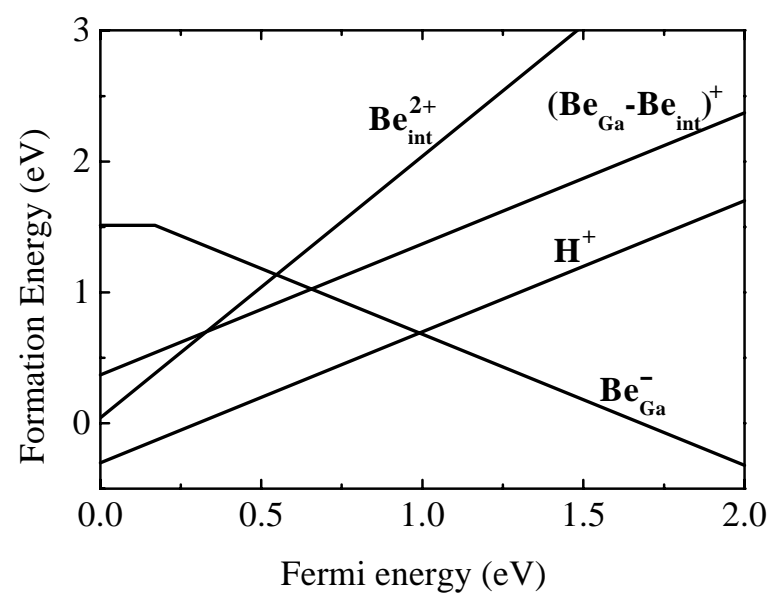

Figure 1. Calculated formation energies as a function of Fermi level for various configurations of Be impurities in wurtzite GaN, under N-rich conditions. The formation energy of $\mathrm{H}^{+}$(assuming equilibrium with $\mathrm{H}_{2}$ ) is also included.

\section{STABILITY AND DIFFUSION OF Be IN GaN}

The equilibrium concentration of an impurity in the semiconductor is determined by its formation energy [4]. Formation energies for various configurations of $\mathrm{Be}$ in $\mathrm{GaN}$ are shown in Fig. 1 as a function of Fermi level. Nitrogen-rich conditions were chosen because they are most favorable for incorporating the $\mathrm{Be}$ acceptor on a Ga site, and equilibrium with $\mathrm{Be}_{3} \mathrm{~N}_{2}$ was assumed. Note that the formation energies of $\mathrm{Be}_{\mathrm{int}}$ and $\mathrm{Be}_{\mathrm{Ga}}$ are comparable under $p$-type conditions. Incorporation of $\mathrm{Be}_{\text {int }}$ is deleterious to $p$-type doping since $\mathrm{Be}_{\text {int }}$ acts as a double donor. We have studied the diffusion behavior of $\mathrm{Be}_{\text {int }}$, in order to assess whether these interstitials are mobile during the growth, and whether it is possible to find a process to get rid of them after growth. For diffusion along the $c$-axis, Be moves through the open channels of the wurtzite structure. Along this path, $\mathrm{Be}$ forms bonds with three $\mathrm{N}$ atoms in the minimum-energy configuration; these bonds are broken in the saddle-point configuration, causing the diffusion barrier to be as large as $2.9 \mathrm{eV}$. On the other hand, for diffusion in the planes perpendicular to the $c$-axis, Be moves from one open channel to an adjacent one, keeping at least two bonds with $\mathrm{N}$ along the entire path; this results in a much lower barrier of $1.2 \mathrm{eV}$. These results suggest that $\mathrm{Be}_{\text {int }}$ should diffuse easily in the (0001) plane, but not along the $c$ axis. Moreover, $\mathrm{Be}_{\text {int }}$ likes to bind with $\mathrm{Be}_{\mathrm{Ga}}$, forming $\left(\mathrm{Be}_{\mathrm{int}}-\mathrm{Be}_{\mathrm{Ga}}\right)$ complexes with a binding energy of $1.35 \mathrm{eV}$. We estimate the dissociation energy of these complexes by adding the (in-plane) migration barrier to the binding energy, yielding a dissociation barrier of $2.55 \mathrm{eV}$.

Hydrogen is known to play an important role in $p$-type doping of GaN [6]. Hydrogen is a donor in $p$-type $\mathrm{GaN}$ and has a high solubility, causing it to readily incorporate during growth. In addition, $\mathrm{Be}_{\mathrm{Ga}} \mathrm{-} \mathrm{H}$ complexes can form with a large binding energy $(1.8 \mathrm{eV}$, compared to 0.7 $\mathrm{eV}$ for $\mathrm{Mg}-\mathrm{H}$ [6]). Incorporating $\mathrm{H}$ during growth will suppress incorporation of $\mathrm{Be}_{\text {int }}$, and simultaneously increase incorporation of $\mathrm{Be}_{\mathrm{Ga}}$. In order to activate the acceptors, hydrogen needs to be removed from the vicinity of the acceptors during a post-growth anneal. We estimate that the $\mathrm{Be}_{\mathrm{Ga}}-\mathrm{H}$ complex has a dissociation barrier of $2.5 \mathrm{eV}$; typical activation anneal temperatures should therefore suffice. These results are discussed in greater detail elsewhere [5]. The presence of acceptor-hydrogen complexes can be assessed by monitoring the local vibration modes associated with these complexes [7]. In the next section we present a detailed account of our investigations of these modes. 


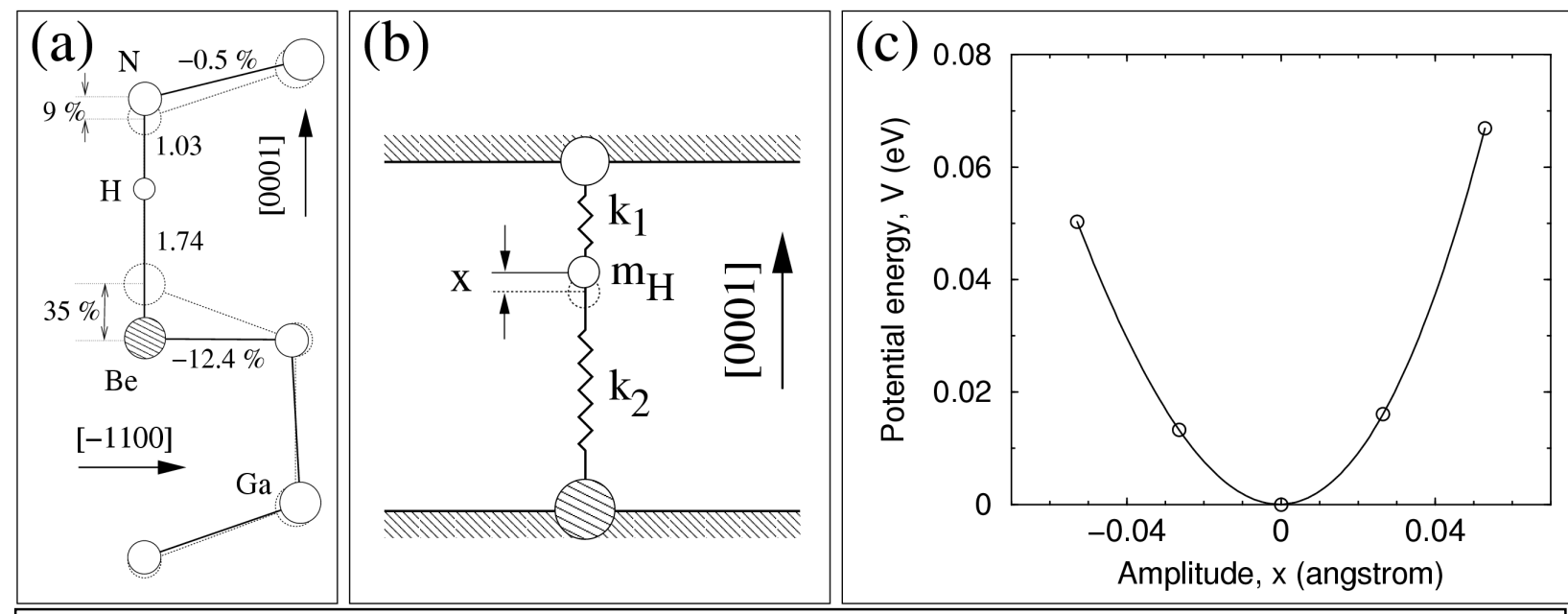

Figure 2. (a) Schematic illustration of the atomic structure of the Be-H complex. Dashed lines represent the ideal structure. Bond lengths are in $\AA$, or indicated as a percentage deviation from the ideal Ga- $N$ bond length. (b) Schematic illustration of the model in which only H moves. (c) Potential energy as a function of the amplitude, $x$.

\section{LOCAL VIBRATION MODES OF Be $\mathrm{Ga}_{\mathrm{G}}-\mathrm{H}$ COMPLEX DEFECT IN GaN}

We have found that the most stable configuration for the $\mathrm{Be}_{\mathrm{Ga}}-\mathrm{H}$ complex has $\mathrm{H}$ sitting in a bond-center (BC) position in a $\mathrm{Be}_{\mathrm{Ga}}-\mathrm{N}$ bond along the $c$ axis [5]. The relaxed structure of the $\mathrm{Be}_{\mathrm{Ga}}-\mathrm{H}$ complex defect in $\mathrm{GaN}$ is shown in Fig 2(a). We set out to calculate the local vibration modes of this complex. By symmetry we can decouple the mode parallel to [0001] (stretch mode) from the mode perpendicular to it (wag mode).

\section{Bond-stretch mode}

To calculate the vibrational frequencies we displace the atoms slightly away from their equilibrium positions and evaluate the energy difference. We perform these calculations at two levels of sophistication. The simplest approach for calculating the vibrational modes is to assume that, since $\mathrm{H}$ is much lighter than any of the other atoms, the surrounding Be and host atoms are fixed in place. The schematic illustration of this model is shown in Fig 2(b). The interatomic interactions are replaced by two springs with force constants $k_{1}$ and $k_{2}$. In this model, the potential and kinetic energies are defined as $V=\frac{1}{2}\left(k_{1}+k_{2}\right) x^{2}$, and $T=\frac{1}{2} m_{\mathrm{H}} \dot{x}^{2}$. To obtain the potential energy as a function of displacement $x$, we perform total energy calculations, using the equilibrium structure as the reference potential energy $(V=0)$. Then we displace the $\mathrm{H}$ atom vertically from the equilibrium position and calculate the total energy, all other atoms remaining fixed. $V(x)$ values from five calculations are shown in Fig 2(c). The deviation from a simple parabola is clear, indicating significant anharmonicity. We fit the data points with a third-degree polynomial with zero slope at the equilibrium position and extract the second-order coefficient $(335.28 \mathrm{~N} / \mathrm{m})$. This gives $\left(k_{1}+k_{2}\right)=670.57 \mathrm{~N} / \mathrm{m}$. Within the simple harmonic model, the relation between the frequency and force constants is: $\omega=\sqrt{\left(k_{1}+k_{2}\right) / m_{\mathrm{H}}}$. Using this relation we get $1 / \lambda=\omega / 2 \pi c=3358 \mathrm{~cm}^{-1}$. This value is quite close to values for the stretch mode of $\mathrm{H}$ in $\mathrm{NH}_{3}$, showing that the $\mathrm{N}-\mathrm{H}$ bond dominates the character of the vibration.

In the simplest approximation, described above, we assumed that only the light $\mathrm{H}$ atoms were allowed to move. However, since the masses of $\mathrm{N}$ and Be are only 14 and 9 times larger than $\mathrm{H}$, it might be important to include the nearest $\mathrm{N}$ and $\mathrm{Be}$ in the model to obtain accurate 


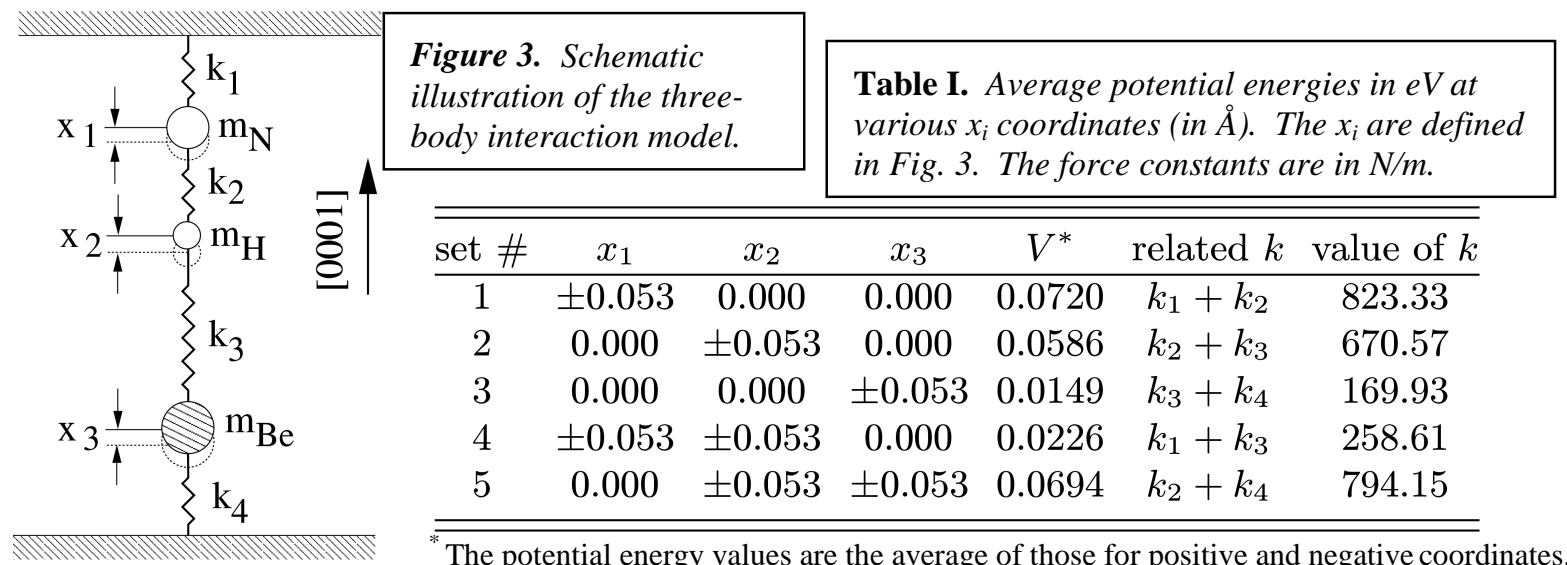

vibrational frequencies. The schematic of this three-body interaction model is shown in Figure 3. The model is similar to that of the free vibrations of a linear triatomic molecule [8] with slight modifications. The interatomic potentials are approximated by four springs with force constants $k_{1-4}$. In these coordinates the potential energy is defined as:

$$
V=\frac{1}{2} k_{1} x_{1}^{2}+\frac{1}{2} k_{2}\left(x_{1}-x_{2}\right)^{2}+\frac{1}{2} k_{3}\left(x_{2}-x_{3}\right)^{2}+\frac{1}{2} k_{4} x_{3}^{2}=x_{1}^{2} \frac{k_{1}+k_{2}}{2}+x_{2}^{2} \frac{k_{2}+k_{3}}{2}+x_{3}^{2} \frac{k_{3}+k_{4}}{2}-x_{1} x_{2} k_{2}-x_{2} x_{3} k_{3} .
$$

To obtain $k_{1-4}$, we perform first-principles calculations for five different sets of coordinates. Each set includes two calculations, one for a positive value of the displacement, the other for a negative value; the average of the two energies is used in the fit, which eliminates the nonsymmetric anharmonic part. The results are tabulated along with the related force constants in Table I. By solving the resulting set of linear equations, we obtain: $k_{1}=205.69 \mathrm{~N} / \mathrm{m}, k_{2}=617.65$ $\mathrm{N} / \mathrm{m}, k_{3}=52.93 \mathrm{~N} / \mathrm{m}$, and $k_{4}=117.01 \mathrm{~N} / \mathrm{m}$. This immediately confirms that $\mathrm{H}$ forms a strong bond with $\mathrm{N}$, indicated by the fact that $k_{2}$ is much larger than the other force constants.

The kinetic energy is

$$
T=\frac{1}{2} m_{\mathrm{N}} \dot{x}_{1}^{2}+\frac{1}{2} m_{\mathrm{H}} \dot{x}_{2}^{2}+\frac{1}{2} m_{\mathrm{Be}} \dot{x}_{3}^{2} .
$$

We define the potential and kinetic matrices, $\mathbf{V}$ and $\mathbf{T}$, by $V=\frac{1}{2} \tilde{\mathbf{x}} \mathbf{V} \mathbf{x}$ and $T=\frac{1}{2} \tilde{\mathbf{x}} \mathbf{T} \dot{\mathbf{x}}$. Then:

$$
\mathbf{V}=\left[\begin{array}{ccc}
k_{1}+k_{2} & -k_{2} & 0 \\
-k_{2} & k_{2}+k_{3} & -k_{3} \\
0 & -k_{3} & k_{3}+k_{4}
\end{array}\right] \text {, and } \mathbf{T}=\left[\begin{array}{ccc}
m_{\mathrm{N}} & 0 & 0 \\
0 & m_{\mathrm{H}} & 0 \\
0 & 0 & m_{\mathrm{Be}}
\end{array}\right] \text {. }
$$

By combining these two matices, the secular equation appears as

$$
\left|\mathbf{V}-\omega^{2} \mathbf{T}\right|=\left|\begin{array}{ccc}
k_{1}+k_{2}-\omega^{2} m_{\mathrm{N}} & -k_{2} & 0 \\
-k_{2} & k_{2}+k_{3}-\omega^{2} m_{\mathrm{H}} & -k_{3} \\
0 & -k_{3} & k_{3}+k_{4}-\omega^{2} m_{\mathrm{Be}}
\end{array}\right|=0 .
$$

By substituting $k_{1-4}$ and masses into above equation and solving the third degree polynomial equation, we obtain $1 / \lambda=\omega / 2 \pi c=475 \mathrm{~cm}^{-1}, 611 \mathrm{~cm}^{-1}$, and $3461 \mathrm{~cm}^{-1}$. The highest frequency is only $3 \%$ different from the value obtained from the simple model above, in which $\mathrm{N}$ and $\mathrm{Be}$ were kept immobile. Note that this $3 \%$ difference can be almost entirely attributed to the choice of reduced mass in the calculation. In our simplest model, we allowed only $\mathrm{H}$ to move, and used only the hydrogen mass in our evaluation of the frequency: $\omega=\sqrt{k / m}$, where $m=m_{\mathrm{H}}$. If, instead, we use the reduced mass for the $\mathrm{N}-\mathrm{H}$ unit (guided by our three-body interaction model showing 

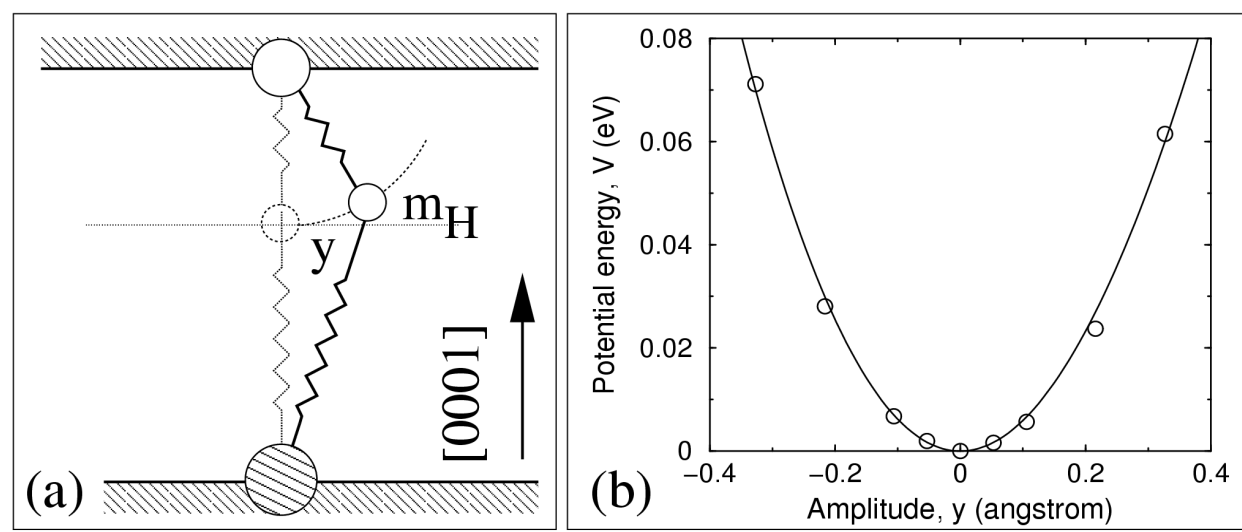

Figure 4.

(a) Schematic illustration of the model for the wag mode of the $\mathrm{Be}-\mathrm{H}$ complex. (b) Potential energy as a function of the amplitude, $y$.

that the high-frequency mode is dominated by the N-H interaction), we obtain $m=0.933 m_{\mathrm{H}}$. Combined with the force constant from our simplest model (only $\mathrm{H}$ moves), we find an increase in the frequency by just over $3 \%$, from $3358 \mathrm{~cm}^{-1}$ to $3476 \mathrm{~cm}^{-1}$, i.e., very close to the result from our more elaborate model.

It is interesting to inspect the characteristics of the other two vibrational modes. The normal mode vectors can be obtained by solving the matrix equation: $\left(\mathbf{V}-\omega^{2} \mathbf{T}\right) \mathbf{A}=\mathbf{0}$, where $\mathbf{A}$ contains the normal mode vector. By substituting each $\omega$ into this equation, we get the normal mode vector for each frequency. The resulting normal mode vectors are $(0.196,0.202,0.216)$ for $475 \mathrm{~cm}^{-1},(0.170,0.141,-0.253)$ for $611 \mathrm{~cm}^{-1}$, and $(-0.068,0.976,-0.010)$ for $3461 \mathrm{~cm}^{-1}$. In mode 1 , all three atoms are moving together at almost the same amplitude. In mode $2, \mathrm{~N}$ and $\mathrm{H}$ move together against $\mathrm{Be}$. And the last mode is an almost pure $\mathrm{H}$ vibration, explaining why the simplest approximation worked so well in this case.

\section{Wag mode}

Our calculations of the stretch mode indicated that the simple approximation in which only $\mathrm{H}$ is allowed to move works very well for the system under study. We therefore use the same approximation here. The model is illustrated in Fig. 4(a). Because the force constant for this mode is much smaller than for the stretch mode, larger displacement amplitudes are needed to obtain reliable potential energies from the first-principles calculations. Our calculations with the three-body interaction model also indicated that the bond between $\mathrm{H}$ and $\mathrm{Be}$ is much weaker than the bond between $\mathrm{H}$ and $\mathrm{N}\left(k_{2}>k_{3}\right)$. In our model we therefore assume that the path of vibration is along the arc shown in Fig 4(a), along which the distance between $\mathrm{H}$ and $\mathrm{N}$ is kept fixed. The amplitude $y$ is defined as the distance by along the arc measured from the equilibrium position. The interatomic interactions are represented with a single force constant, $k$. We perform first-principles calculations for nine $y$ values up to amplitudes as large as $0.4 \AA$; the results are shown along with the polynomial fit in Fig 4(b). From the fit we obtain $k=19.52 \mathrm{~N} / \mathrm{m}$, and $1 / \lambda=573 \mathrm{~cm}^{-1}$.

\section{DISCUSSION AND CONCLUSIONS}

We have reported first-principles results for Be impurities in GaN. Beryllium appears to be a better acceptor than $\mathrm{Mg}$ in $\mathrm{GaN}$ for two reasons [4,5]. First, the formation energy of $\mathrm{Be}$ acceptors is slightly lower than that of $\mathrm{Mg}$, indicating higher solubility. Second, Be has a slightly lower ionization energy than $\mathrm{Mg}$. We also find that Be interstitials, which act as double donors, have a low formation energy hence are likely to incorporate during growth. We studied the diffusion of Be interstitials and found highly anisotropic behavior. The diffusion along the $c$ 
axis has the barrier of $2.9 \mathrm{eV}$, which is much larger than that of the in-plane diffusion perpendicular to the $c$ axis $(1.2 \mathrm{eV})$. Beryllium interstitials may bind to substitutional acceptors, with a binding energy of $1.35 \mathrm{eV}$ and a dissociation energy of $2.55 \mathrm{eV}$.

We suggest that incorporating $\mathrm{H}$ during growth would suppress Be interstitials. Berylliumhydrogen complexes may form with a binding energy of $1.8 \mathrm{eV}$ and a dissociation energy of 2.5 $\mathrm{eV}$. These Be-H complexes have the lowest formation energy of all defects discussed here which means these complexes have very high solubility. A post-growth anneal would remove $\mathrm{H}$ from the complexes, activating the Be acceptors. The presence of Be-H complexes can be probed by monitoring their local vibrational modes. We have explained in detail our first principles calculations of bond-stretch and wag modes. Based on the numerical accuracy of the calculations and on the inherent accuracy of the methods, we estimate the error bar on our calculated frequencies to be about $100 \mathrm{~cm}^{-1}$. For the bond-stretch mode we find a frequency of $3461 \mathrm{~cm}^{-1}$. This value is close to vibrational stretch frequencies in $\mathrm{NH}_{3}$ molecules. It is also close to the calculated stretch mode in $\mathrm{Mg}-\mathrm{H}$ complexes [6], in spite of the fact that the equilibrium structure of $\mathrm{Mg}-\mathrm{H}$ complexes is different from $\mathrm{Be}-\mathrm{H}$. In $\mathrm{Mg}-\mathrm{H}, \mathrm{H}$ occupies an antibonding position behind a $\mathrm{N}$ atom, while in $\mathrm{Be}-\mathrm{H}, \mathrm{H}$ sits in a $\mathrm{BC}$ site. However, given our finding that the frequency is dominated by the $\mathrm{N}-\mathrm{H}$ interaction, the similarity in the frequencies is not surprising. Note that we did not include anharmonic effects [9], which are expected to be sizeable for vibrations involving the light $\mathrm{H}$ atom. In $\mathrm{NH}_{3}$ the anharmonicity lowers the symmetric stretch frequency by $170 \mathrm{~cm}^{-1}$ [10]. To extract accurate values for the anharmonic effect one would need to map out the potential energy surface in greater detail. This will be the subject of a future investigation. For the wag mode of the Be-H complex, finally, the restoring force is much smaller than the bond-stretch mode. We obtained a frequency of $573 \mathrm{~cm}^{-1}$.

\section{ACKNOWLEDGMENTS}

This work was supported by the Office of Naval Research, Contract No. N00014-99-C0161, monitored by C. Wood. J.N. thanks the German Science Foundation (Schwerpunktprojekt Gruppe-III-Nitride) for financial support. We thank J. Northrup, L. Romano, N. Johnson, M. Kneissl, P. Kiesel, W. R. L. Lambrecht, and T. H. Myers for useful discussions.

\section{REFERENCES}

1. D. P. Bour, H. F. Chung, W. Götz, L. Romano, B. S. Krusor, D. Hofstetter, S. Rudaz, C. P. Kuo, F. A. Ponce, N. M. Johnson, M. G. Craford, and R. D. Bringans, Mater. Res. Soc. Symp. Proc. 449, 509 (1997).

2. W. Götz, R. S. Kern, C. H. Chen, H. Liu, D. A. Steigerwald, and R. M. Fletcher, Mater. Sci. Engin. B 59, 211 (1999).

3. F. Bernardini, V. Fiorentini, and A. Bosin, Appl. Phys. Lett. 70, 2990 (1997).

4. J. Neugebauer and C. G. Van de Walle, J. Appl. Phys. 85, 3003 (1999).

5. C. G. Van de Walle, S. Limpijumnong, and J. Neugebauer, "First-principles studies of beryllium doping of GaN”, submitted to Phys. Rev. B.

6. J. Neugebauer and C. G. Van de Walle, Phys. Rev. Lett. 75, 4452 (1995).

7. W. Götz, N. M. Johnson, D. P. Bour, M. D. McCluskey, and E. E. Haller, Appl. Phys. Lett. 69, 3725 (1996).

8. H. Goldstein, Classical Mechanics (Addison-Wesley, Philippines, 1980).

9. C. G. Van de Walle, Phys. Rev. Lett. 80, 2177 (1998).

10. B. G. Johnson, P. M. W. Gill, and J. A. Pople, J. Chem. Phys. 98, 5612 (1993). 